\title{
A novel approach for monitoring tobacco use in local communities by wastewater analysis
}

\author{
Sara Castiglioni, ${ }^{1}$ Ivan Senta, ${ }^{2}$ Andrea Borsotti, ${ }^{1}$ Enrico Davoli, ${ }^{1}$ Ettore Zuccato ${ }^{1}$
}

\begin{abstract}
${ }^{1}$ Department of Environmental Health Sciences, IRCCSIstituto di Ricerche Farmacologiche "Mario Negri", Milan, Italy

${ }^{2}$ Division for Marine and Environmental Research, Rudjer Boskovic Institute, Zagreb, Croatia
\end{abstract}

\section{Correspondence to}

Dr Sara Castiglioni,

Department of Environmental

Health Sciences, IRCCS-Istituto

di Ricerche Farmacologiche

"Mario Negri", Via La Masa

19, Milan 20156, Italy;

sara.castiglioni@marionegri.it

Received 9 January 2014

Accepted 18 June 2014

Published Online First

11 July 2014

\section{SLinked}

- http://dx.doi.org/10.1136/ tobaccocontrol-2014-051844

\section{CrossMark}

$$
\begin{aligned}
& \text { To cite: Castiglioni S, } \\
& \text { Senta I, Borsotti A, et al. } \\
& \text { Tob Control 2015;24: }
\end{aligned}
$$$$
\text { 38-42. }
$$

\section{ABSTRACT}

Objective We propose a novel approach for measuring tobacco use in a community through the chemical analysis of nicotine metabolites in urban wastewater. It offers frequent monitoring and 'real-time', 'evidencebased' estimates of tobacco consumption which may complement epidemiological surveillance systems normally repeated only every few years.

Methods Two urinary metabolites of nicotine, namely cotinine and trans-3'-hydroxycotinine, were selected as biomarkers of tobacco consumption in urban wastewater. During smoking, a known amount of nicotine is absorbed and after metabolism excreted as metabolites in urine, ending up in the wastewater; quantitative analysis of the metabolites in the wastewater allows back-calculation of the nicotine collectively absorbed by the population producing the sewage and, indirectly, their tobacco use. Representative samples of wastewater were collected from sewage treatment plants in eight Italian cities and analysed by mass spectrometry. Mass loads of the metabolites were used to estimate nicotine consumption.

Results Wastewater analysis in the cities under study was used to estimate the number of cigarettes smoked, in order to compare the results of this study with those obtained from population surveys. The number of cigarettes calculated with the two methods were closely comparable and wastewater analysis was sufficiently sensitive to confirm the differences in tobacco consumption between northern and southern Italy, previously described in population surveys.

Conclusions The described approach can serve as a supplementary indicator of tobacco consumption in local communities. This approach can provide objective and updated information, which are useful to assess the efficacy of tobacco-control interventions, with the aim of designing and implementing effective tobacco control plans.

\section{INTRODUCTION}

A certain amount of the nicotine that is absorbed by the body during smoking is excreted in urine as the parent drug (nicotine) and the products of metabolism (metabolites), mainly cotinine and trans-3'-hydroxycotinine. ${ }^{1}$ This urine then enters the wastewater in the sewerage network. The wastewater can therefore be seen as a 'depository' for any sufficiently stable compound excreted by the local population. ${ }^{2}$ Thus, measuring the excretion products of nicotine in wastewater can serve to back-calculate the nicotine absorbed (NICabs), and indirectly assess tobacco smoking by the population producing the sewage. If regularly monitored, changing mass loads of the residues in wastewater could reflect changes in smoking giving updated and objective information of population level nicotine consumption. This information can complement survey and cigarette sales data by providing population cigarette use estimates that are not affected by misreporting of smoking status, underestimation of cigarette use ${ }^{3}$ or cigarette smuggling. ${ }^{4}$

Smoking is the first preventable cause of premature mortality worldwide ${ }^{5}$ and accounts for $12.5 \%$ of total mortality in Italy, ${ }^{6}$ thus an integrative method to monitor cigarette use in a certain population can also be useful to design preventive campaigns at a local level and evaluate the impact of tobacco control strategies.

As an example of this approach, the chemical analysis of wastewater has been used to estimate illicit drug consumption ${ }^{7}$ and alcohol intake ${ }^{8}$ in local communities. The European Monitoring Centre for Drugs and Drug Addiction (EMCDDA) has recognised the potential of this methodology to complement and extend the existing epidemiologically based illicit drug use estimation techniques. This approach has been called 'sewage epidemiology" ${ }^{7}$ or, recently, "wastewater-based epidemiology', and the analysis of a broad list of sewage biomarkers has been proposed to public health agencies for quantitative measurement of the health of a population. ${ }^{10}$ To be used for this purpose, these biomarkers need to be sufficiently stable to survive the journey from toilet to sampling point (mean residence time of sewage in the sewer systems $0.5-15 \mathrm{~h}),{ }^{11}$ be specific for the substance used as urinary excretion products, and be excreted at sufficient concentrations to be detected. Additionally, the excretion profile of the biomarkers must be known to develop proper correction factors for back-calculating the consumption of the substance used.

This study shows that two human metabolic products of nicotine, cotinine and trans-3'-hydroxycotinine, can be measured in urban wastewater, and fulfil all the requirements for use as sewage biomarkers for estimating collective consumption of tobacco in communities, with the unique potential to monitor local consumption trends in real time.

\section{METHODS}

Nicotine and its human metabolic residues were measured in urban wastewater by high-performance liquid chromatography tandem mass spectrometry. This technique was chosen because it is very sensitive and specific for quantifying traces of the selected analytes even in a complex matrix such as raw urban wastewater. The analytical method was modified from previous publications ${ }^{12} 13$ and was validated according to the required good practice 
criteria in order to ensure high quality and repeatability of results.

Twenty-four hour composite samples of raw wastewater were collected daily for several consecutive days, at the entry to some sewage treatment plants (STPs), each serving a different Italian city (Milan, Como, Bologna and Turin in northern Italy, Rome in central Italy, and Naples, Bari and Palermo in southern Italy). One-week sampling was performed in October 2012 contemporaneously in all the cities investigated.

Each STP collects almost the whole amount of urban wastewater produced in a city, thus being representative of the population of that city. Sampling followed the current best practice protocol to obtain non-biased composite samples and was performed using a volume-proportional mode. ${ }^{11}$

Samples were filtered, enriched with labelled (deuterated) internal standards, and solid phase extracted using mixed reverse-phase cation exchange cartridges (Oasis MCX, Waters Corp, Milford, Massachusetts, USA). A triple quadrupole mass spectrometer (AB SCIEX QqQ 5500, Ontario, Canada) was used for analyses of the selected analytes. Quantification was performed using analogue labelled standards for each compound, thus enabling a higher performance of the method and the correction for matrix effects. The stability of the compounds in the sewer network and during storage and analyses was also checked experimentally and the analytes were stable in all the conditions $\left(24 \mathrm{~h}\right.$ at $4^{\circ} \mathrm{C}$ and $20^{\circ} \mathrm{C}$, and 28 days at $-20^{\circ} \mathrm{C}$ ). Triplicate analyses have been performed for each condition and no losses of these compounds were observed during the experiment (recovery of analytes 96-104\% $02-6 \%$-relative SD). Measured concentrations of nicotine and metabolites (ng/L) were multiplied by the daily flow rate of wastewater $\left(\mathrm{m}^{3} /\right.$ day) at the entry of the treatment plant, to obtain a daily mass load for each substance (g/day).

Nicotine human excretion studies were analysed to develop specific correction factors to back-calculate nicotine intake by the population, starting from the metabolic residues measured in raw wastewater. From the available literature ${ }^{114} 15$ we identified the mean percentages of excretion of cotinine and trans-3'-hydroxycotinine after nicotine absorption (table 1 ). The conjugated forms of these metabolites are completely transformed to the free form by $\beta$-glucuronidase enzymes from faecal bacteria in the raw wastewater. ${ }^{16}{ }^{17}$ The excretion rates of these two metabolites ( $\%$ of free and conjugated forms) were summed and the mean (74\%) was used to develop a correction factor. Considering this, for $1 \mathrm{mg}$ of NICabs during smoking, the excretion of nicotine equivalents is $0.74 \mathrm{mg}$, hence the correction factor was calculated as follows: $(1 / 0.74)=1.35$. The mass load of the parent drug (nicotine) in the wastewater was not considered in the back-calculation, as sources other than

Table 1 Metabolic profile of nicotine excretion in human urine according to the literature

\begin{tabular}{|c|c|c|c|}
\hline Investigated substances & Nicotine & Cotinine & $\begin{array}{l}\text { trans- } 3^{\prime}- \\
\text { hydroxycotinine }\end{array}$ \\
\hline Human excretion (\%)—free form* & 10 & 13 & 35 \\
\hline $\begin{array}{l}\text { Human excretion }(\%) \text {-conjugated } t \\
\text { form* }\end{array}$ & 3 & 17 & 9 \\
\hline Sum free+conjugated forms (\%) & 13 & 30 & 44 \\
\hline
\end{tabular}

human excretion, such as flushing of cigarette ash or cigarettes down toilets, can contribute to its occurrence in wastewater. The NICabs (g/day) was obtained through the following equation:

$$
\text { NICabs }=[(\mathrm{C} \cot \times \mathrm{F})+(\text { Chy } \cot \times \mathrm{F})] \times \mathrm{Cf}
$$

where C cot and Chy cot are, respectively, the concentration of cotinine and trans-3'-hydroxycotinine measured in wastewater $(\mathrm{ng} / \mathrm{L}), \mathrm{F}$ is the flow rate of wastewater $(\mathrm{L} /$ day), Cf the correction factor (1.35).

To compare these results with those of epidemiological surveys, we used NICabs (the estimate of total nicotine absorbed by the population under study) to calculate the number of cigarettes consumed, considering that during smoking for one cigarette an average of $1.25 \mathrm{mg}$ of nicotine is absorbed systemically. ${ }^{1}$ Official prevalence data from one epidemiological survey conducted in 2012 by DOXA, the Italian branch of the Worldwide Independent Network/Gallup International Association (WIN/ GIA) were also used to calculate the number of cigarettes smoked. This survey had a sample size of more than 3000 individuals, representative of the general population aged 15 years or over in terms of age, sex, geographic area and socioeconomic characteristics. ${ }^{18}$ Prevalence data were used to calculate the number of smokers considering the population aged $>14$ years in each city (obtained from the Italian National Institute of Statistics), ${ }^{19}$ and to estimate the number of cigarettes using the average number consumed daily by a smoker in Italy. ${ }^{18}$ Finally, the number of cigarettes estimated through wastewater analysis and survey data were compared calculating the difference as number of cigarettes and percentage.

\section{RESULTS}

Figure 1 reports an 18-day profile of excretion of nicotine, cotinine and trans-3'-hydroxycotinine in wastewater in Milan. Cotinine and trans-3'-hydroxycotinine mass loads in wastewater (about 500 and $1000 \mathrm{~g} /$ day, respectively) were as expected, considering the excretion in urine (table 1), and their levels were

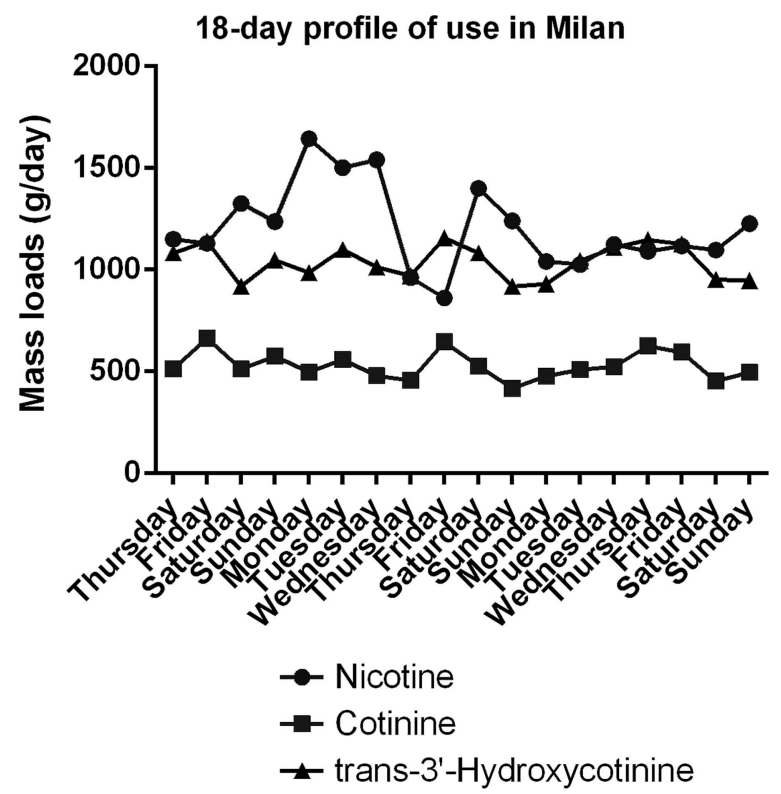

Figure 1 Eighteen-day profile of excretion of nicotine, cotinine and trans-3'-hydroxycotinine in wastewater in Milan. Means of two sampling campaigns, September and December 2012. 
Table 2 Comparison among the number of cigarettes smoked estimated through wastewater analysis and epidemiological surveys in the population investigated

\begin{tabular}{|c|c|c|c|c|c|c|c|c|}
\hline \multirow[b]{2}{*}{$\begin{array}{l}\text { Cities } \\
\text { investigated }\end{array}$} & \multicolumn{3}{|l|}{ Wastewater analysis } & \multicolumn{3}{|c|}{ Epidemiological survey data } & \multicolumn{2}{|c|}{$\begin{array}{l}\text { Difference in the number of cigarettes } \\
\text { smoked }\end{array}$} \\
\hline & $\begin{array}{l}\text { Population } \\
\text { (>14 years) served } \\
\text { by the STP* }\end{array}$ & $\begin{array}{l}\text { Estimated NICabs } \\
\text { (g/day; mean } \\
\pm \text { SD) } \dagger\end{array}$ & $\begin{array}{l}\text { Number of } \\
\text { cigarettes } \\
\text { smoked } \neq\end{array}$ & $\begin{array}{l}\text { Prevalence of } \\
\text { smokers } \\
\text { ( } \% \text { of population } \\
\geq 15 \text { years) }\end{array}$ & $\begin{array}{l}\text { Number of } \\
\text { smokers§ }\end{array}$ & $\begin{array}{l}\text { Number of } \\
\text { cigarettes } \\
\text { smokedๆ }\end{array}$ & $\begin{array}{l}\text { Number of } \\
\text { cigarettes } \\
\text { (survey } \\
\text { data-wastewater } \\
\text { analysis results) }\end{array}$ & $\begin{array}{l}\text { Percentage (survey } \\
\text { data-wastewater } \\
\text { analysis results) }\end{array}$ \\
\hline \multicolumn{9}{|l|}{ Northern Italy } \\
\hline Milan & 960300 & $2121 \pm 237$ & 1696800 & 19.6 & 188219 & 2409201 & +712401 & +30 \\
\hline Como & 79926 & $241 \pm 51$ & 192800 & 19.6 & 15665 & 200518 & +7718 & +4 \\
\hline Bologna & 470640 & $1360 \pm 127$ & 1087726 & 19.6 & 92245 & 1180742 & +93016 & +8 \\
\hline Turin & 1201490 & $4756 \pm 393$ & 3804828 & 19.6 & 235492 & 3014298 & -790530 & -26 \\
\hline Mean & & & 1695538 & & & 1701190 & +5651 & +0.3 \\
\hline \multicolumn{9}{|l|}{ Central Italy } \\
\hline Rome & 1107699 & $3734 \pm 1779$ & 2987243 & 19 & 210463 & 3114850 & +127606 & +4 \\
\hline \multicolumn{9}{|l|}{ Southern Italy } \\
\hline Naples & 549250 & $2492 \pm 266$ & 1993948 & 23.3 & 127975 & 1714868 & -279080 & -16 \\
\hline Bari & 296922 & $1076 \pm 43$ & 860912 & 23.3 & 69183 & 927050 & +66137 & +7 \\
\hline Palermo & 223254 & $958 \pm 75$ & 766416 & 23.3 & 52018 & 697044 & -69372 & -10 \\
\hline Mean & & & 1207092 & & & 1112987 & -94105 & -8 \\
\hline
\end{tabular}

Bold denotes the mean values.

*The total population (served by the STP) was corrected by the percentage of people $<14$ years. 19

tMean and SD are calculated from the daily mass loads obtained for each city. Sampling campaigns were: 18 days in Milan and 7 days in all the other cities. ¥Calculated using the mean content of nicotine absorbed from one cigarette $(1.25 \mathrm{mg})^{.}{ }^{1}$

$\S$ Calculated from prevalence data considering the population served by the STPs investigated.

TCalculated considering the average number of cigarettes smoked per day (12.8 86.8 in the North of Italy, $14.8 \pm 6.7$ in the Center and $13.4 \pm 7.7$ in the South) ${ }^{18}$

NICabs, nicotine absorbed; STP, sewage treatment plant. 
stable over the whole sampling period (figure 1). In contrast, parent nicotine mass loads (about $1200 \mathrm{~g} /$ day) were higher than expected considering only urinary excretion (table 1) and showed random variability during the sampling period. This may indicate that other sources of nicotine, such as direct disposal of ash and wash out from cigarette butts, contribute to the amount of nicotine in wastewater. This is the reason why we decided to rely on human metabolites in wastewater and not on nicotine itself to estimate the intake by the population.

Table 2 reports the comparison among the number of cigarettes smoked estimated through wastewater analysis and epidemiological surveys. Nicotine intakes (NICabs) were estimated by back-calculation, the number of cigarettes smoked was then calculated for each city and ranged between 190000 and 3805000 depending on the size of the population. The number of cigarettes smoked was also calculated for each city from the prevalence of smokers estimated by epidemiological surveys in northern, central and southern Italy. The results obtained from wastewater analysis matched those from prevalence data. In the North of Italy four cities were investigated (Milan, Como, Bologna and Turin) giving a mean difference of the number of cigarettes smoked as 5651 cigarettes $(0.3 \%)$. In central Italy only Rome was investigated and the mean difference of the number of cigarettes smoked was 127606 (4\%). In the South of Italy three cities were investigated (Naples, Bari and Palermo), and the mean difference of the number of cigarettes smoked was $94105(8 \%)$. Therefore the results obtained from wastewater analysis matched the prevalence from population surveys and confirmed epidemiological reports, indicating a higher prevalence of smoking in southern than northern and central Italy.

\section{DISCUSSION}

Accurate monitoring of tobacco use is essential to evaluate the impact of tobacco-control policies. ${ }^{20}$ However, fewer than half of European countries conduct regular tobacco use surveys annually or biennially. ${ }^{21}$ The present study reports a novel approach based on the chemical analysis of nicotine excretion products in urban wastewater to evaluate tobacco use in a population. The results show good agreement between the number of cigarettes smoked calculated by wastewater analysis and from epidemiological surveys in Italy. The wastewater analysis approach was applied in several cities in different parts of Italy and the results were in good agreement with those obtained from epidemiological surveys (table 2). The differences in smoking prevalence between the northern-central and the South of Italy, already identified through epidemiological studies, could be confirmed through wastewater analysis. Similar results were obtained in Lisbon, where only cotinine was measured in three STPs to back-calculate nicotine consumption, and results were in line with a European Survey. ${ }^{22}$ This confirms the reliability of the approach for monitoring tobacco use in a population. The strength of the wastewater-analysis approach is its ability to provide 'real-time' up-to-date information on smoking trends in local communities. Moreover, the information can be obtained at lower cost and in a shorter time than epidemiological surveys. However, as suggested for illicit drugs, ${ }^{23}$ this approach makes no claim to replace, but only to complement epidemiological studies by providing a tool for more frequently assessing tobacco use at local levels, thus making it possible to detect and follow changing trends in populations, and to assess the efficacy of tobacco-control strategies.

As described for illicit drugs, ${ }^{7}$ the wastewater approach suffers some sources of uncertainty, which can be ascribed to variability in wastewater sampling, stability of biomarkers in sewage, accuracy of analytical measurements, uncertainty in metabolism and excretion data used for back-calculation, and in estimating population sizes. ${ }^{11}$ All these factors have been recently examined in order to minimise, where possible, the uncertainty of the entire procedure and make the results more reliable. A best practice protocol was developed, considering the latest scientific information ${ }^{11} 23$ and it was proposed to the scientific community to reduce the potential biases of this approach. In the present study, the potential uncertainty factors were carefully checked according to that proposed protocol, to ensure acceptable reliability of results. Specifically, for the adopted sampling, protocol uncertainty was lower than $10 \%,{ }^{11}$ the stability of nicotine excretion products in sewage was evaluated and the analytical method was fully validated keeping the specific uncertainties lower than $10 \%$. Moreover, the pharmacokinetic data in the literature on nicotine metabolism were consistent in different papers, and the correction factor employed to back-calculate from metabolites to nicotine was therefore considered highly reliable.

Another possible confounding factor is the absorption of nicotine through routes other than smoking, such as all nicotine replacement therapies (NRTs): nicotine patches, pills and gums, and the use of electronic cigarettes. The contribution of these products to the total nicotine load was not quantified in this study but can be estimated from sales figures. In Europe, in 2010, e-cigarettes and NTRs each had a $0.4 \%$ share of the European Union (EU) tobacco and nicotine market. ${ }^{24}$ Also, in a recent European Commission publication it has been estimated that among $27 \mathrm{EU}$ countries, 5\% of survey responders have tried e-cigarettes once and only $1 \%$ are occasional or regular user. ${ }^{25}$ As mentioned before, the potential uncertainty of the protocol is below $10 \%$ and the potential estimation error due to NRTs and e-cigarettes is therefore negligible and has not been quantified in this work.

\section{CONCLUSIONS}

To the best of our knowledge this is the first study showing that the metabolites of nicotine excreted in the urine of smokers can be measured in wastewater and that their concentrations can be used to estimate and monitor nicotine absorption and, indirectly, smoking in the communities producing the wastewater. This novel approach to estimate smoking in populations is not intended to replace, but only to complement population surveys, and will provide a tool for assessing tobacco use more frequently than by epidemiological studies, and make it possible to follow in 'real-time' changing trends in populations, and the efficacy of tobacco-control policies.

\section{What this paper adds?}

- This paper describes a novel approach for measuring tobacco use based on wastewater analysis.

- The approach offers frequent monitoring and 'real-time', 'evidence-based' estimates of tobacco consumption.

- The number of cigarettes calculated through population surveys and wastewater analysis were closely comparable.

Acknowledgements All the authors wish to thank Silvano Gallus and Alessandra Lugo (Department of Epidemiology, IRCCS - Istituto di Ricerche Farmacologiche 
"Mario Negri") for providing data on smoking prevalence and daily cigarette consumption in Italy by geographic area and Emma Gracia Lor for revision of the manuscript. Francesca Pizza, Carlo Amadasi (Milano Depur SpA-Milano), Francesca Castelli (Como Depur SpA-Como), Francesco Avolio, (HERA SpABologna), Lorenza Meucci (Società Metropolitana Acque Torino SpA-Turin), Roberto Celestini, Fabrizio De Rosa (Acea AT02 SpA-Rome), Silvana Fiorillo (Impianto di depurazione di Cuma-Naples), Santa Busano (Acquedotto Pugliese SpA-Bari) and Antonella Cicala (AMAP SpA-Palermo) for the technical support during sampling.

Contributors SC designed the study, performed data elaboration and wrote the manuscript; IS and $A B$ performed the analyses and revised the manuscript; $E D$ designed the study and revised the manuscript; and EZ designed the study, performed data elaboration and wrote the manuscript.

Funding The study was supported by Fondazione Cariplo (Grant 2009-3468-2009-3513) and Dipartimento Politiche Antidroga (Presidenza del Consiglio dei Ministri, Rome)—Project Aqua Drugs. Part of this study has received funding from the European Union's Sevenths Framework Programme for research, technological development and demonstration under grant agreement no [317205].

\section{Competing interests None.}

Provenance and peer review Not commissioned; externally peer reviewed.

\section{REFERENCES}

1 Hukkanen J, Jacob P III, Benowitz NL. Metabolism and disposition kinetics of nicotine. Pharmacol Rev 2005;57:79-115.

2 Castiglioni S, Zuccato E, Fanelli R, eds. Illicit drugs in the environment. Occurrence, analysis, and fate using mass spectrometry. Hoboken, NJ: John Wiley\& Sons, 2011.

3 Gallus S, Tramacere I, Boffetta P, et al. Temporal changes of under-reporting of cigarette consumption in population-based studies. Tob Control 2011;20:34-9.

4 Gallus S, Tramacere I, Zuccaro P, et al. Cigarette smuggling in Italy, 2005-8. Tob Control 2009;18:159-60.

5 World Health Organization (WHO). WHO report on global tobacco epidemic. Geneva, Switzerland: World Health Organization, 2013.

6 Gallus S, Muttarak R, Martinez-Sanchez JM, et al. Smoking prevalence and smoking attributable mortality in Italy, 2010. Prev Med 2011;52:434-8.

7 Zuccato E, Chiabrando C, Castiglioni S, et al. Estimating community drug abuse by wastewater analysis. Environ Health Perspect 2008;116:1027-32.

8 Reid MJ, Langford KH, Morland J, et al. Analysis and interpretation of specific ethanol metabolites, ethyl sulfate, and ethyl glucuronide in sewage effluent for the quantitative measurement of regional alcohol consumption. Alcohol Clin Exp Res 2011;35:1593-9.

9 European Monitoring Center for Drugs and Drug Addiction (EMCDDA). Assessing illicit drugs in sewage-Potential and limitations of a new monitoring approach. Luxembourg: European Monitoring Center for Drugs and Drug Addiction, 2008.
10 Thomas KV, Reid MJ. What else can the analysis of sewage for urinary biomarkers reveal about communities? Environ Sci Technol 2011;45:7611-12.

11 Castiglioni S, Bijlsma L, Covaci A, et al. Evaluation of uncertainties associated with the determination of community drug use through the measurement of sewage drug biomarkers. Environ Sci Technol 2013;47:1452-60.

12 Huerta-Fontela M, Galceran MT, Ventura F. Ultraperformance liquid chromatography-tandem mass spectrometry analysis of stimulatory drugs of abuse in wastewater and surface waters. Anal Chem 2007:79:3821-9.

13 Bueno MJ, Ucles S, Hernando MD, et al. Evaluation of selected ubiquitous contaminants in the aquatic environment and their transformation products. A pilot study of their removal from a sewage treatment plant. Water Res 2011:45:2331-41.

14 Byrd GD, Chang KM, Greene JM, et al. Evidence for urinary excretion of glucuronide conjugates of nicotine, cotinine, and trans-3'-hydroxycotinine in smokers. Drug Metab Dispos 1992;20:192-7.

15 Baselt R. Disposition of toxic drugs and chemicals in man. Biomedical Publications, 2004.

16 D'Ascenzo G, Di Corcia A, Gentili A, et al. Fate of natural estrogen conjugates in municipal sewage transport and treatment facilities. Sci Total Environ 2003:302:199-209.

17 Castiglioni S, Zuccato E, Crisci E, et al. Identification and measurement of illicit drugs and their metabolites in urban wastewater by liquid chromatography-tandem mass spectrometry. Anal Chem 2006;78:8421-9.

18 Gallus S, Lugo A, Colombo P, et al. Smoking prevalence in Italy 2011 and 2012, with a focus on hand-rolled cigarettes. Prev Med 2013;56:314-18.

19 Italian National Institute of Statistics (ISTAT). Censimento Popolazione 2011. Rome, Italy, 2011. http://www.istat.it/it/ (accessed 16 Apr 2014).

20 World Health Organization (WHO). WHO report on the global tobacco epidemic 2008: the MPOWER Package. Geneva, Switzerland: World Health Organization, 2008.

21 Bogdanovica I, Godfrey F, McNeill A, et al. Smoking prevalence in the European Union: a comparison of national and transnational prevalence survey methods and results. Tob Control 2011;20:e4.

22 Lopes A, Silva N, Bronze MR, et al. Analysis of cocaine and nicotine metabolites in wastewater by liquid chromatography-tandem mass spectrometry. Cross abuse index patterns on a major community. Sci Total Environ 2014:487:673-80.

23 Castiglioni S, Thomas KV, Kasprzyk-Hordern B, et al. Testing wastewater to detect illicit drugs: state of the art, potential and research needs. Sci Total Environ 2014;487:613-20.

24 Erbach G. Electronic cigarettes. Library briefing. Library of the European Parliament, 2013. http://www.europarl.europa.eu/eplibrary/Electronic-cigarettes.pdf (accessed 16 Apr 2014)

25 TNS Opinion \& Social. Attitudes of Europeans towards tobacco. Special Eurobarometers 385, Wave EB77.1. Directorate General Health and Consumers, European Commission, Brussels 2012 